\title{
Digital in-line holography with a spatially partially coherent beam
}

\author{
S. Coëtmellec \\ coetmellec@coria.fr \\ C. Remacha
}

\section{Brunel \\ D. Lebrun}

\section{A. J. E. M. Janssen}

\begin{abstract}
Departement Optique-Lasers, UMR-6614 CORIA, Av. de l'Universite, 76801 Saint-Etienne du Rouvray, France

Departement Optique-Lasers, UMR-6614 CORIA, Av. de l'Universite, 76801 Saint-Etienne du Rouvray, France

Departement Optique-Lasers, UMR-6614 CORIA, Av. de 1'Universite, 76801 Saint-Etienne du Rouvray, France

Departement Optique-Lasers, UMR-6614 CORIA, Av. de l'Universite, 76801 Saint-Etienne du Rouvray, France

Department of Electrical Engineering and Department of Mathematics and Computer Science, Technische Universiteit Eindhoven, 5600 MB Eindhoven, Netherlands
\end{abstract}

We propose in this paper an analytical solution to the problem of scalar diffraction of a partially coherent beam by an opaque disk. This analytical solution is applied in digital in-line holography of particles. We demonstrate that the reconstruction by means of fractional Fourier transformation is still possible when a spatially partially coherent beam is used. Numerical simulations and experiments have been carried out. [DOI: http://dx.doi.org/10.2971/jeos.2011.11060]

Keywords: Digital in-line holography

\section{INTRODUCTION}

Generally, digital in-line or off-axis holography require a coherent laser source to record a diffraction pattern by means of a CCD sensor [1]. These coherent sources are sensitive to defects in the optical system on one hand and generate unwanted high spatial frequencies in the plane of the CCD sensor on the other hand. It is well known that the interaction of the such beams with the periodic structures of the pixels matrix create Moiré effects. In particular cases, this phenomenon allows to elaborate some metrology techniques [2] but in the case of particle field analysis the Moire fringes are considered here as a drawback in Digital In-line Holography (DIH). Several methods are possible to reduce these effects. Firstly, it is possible to choose an optimal sampling rate of solid-state detectors to eliminate the under-sampling of the hologram (Shannon's criterium). The second possibility is to impose some constraints on the maximum angle between the object beam and the reference wave $[3,4]$. The third and final point concerns the characteristics of the laser source. There are two ways that can be considered in order to adapt the light source so that the above problem is avoided. The first way consists in enlarging the frequency spectrum (linked to the wavelengths) of the laser source to attenuate the high frequencies contained in the recorded hologram. In this context, a natural low-pass filter is applied on the fringe pattern [5]. The second way is to work on the spatial coherence of the source (generally we called visibility function) [6,7]. This paper is devoted to propose an analytical solution to the problem of scalar diffraction of a partially spatially coherent beam by an opaque disk. Recall that the basic idea in digital in-line holography is firstly to record the intensity distribution, with a CCD sensor, of the diffraction pattern of a particle field illuminated by a light wave $[8,9,10]$. The second step is to reconstruct the image of this field by means of an operator [11, 12, 15]. The knowledge of the theoretical model in digital in-line holography allows us to digitally refocus, in an optical sense, on the objects. Consequently, in the following section, the analytical model is given in the case of a partially spatially coherent beam and the theoretical intensity is computed in semianalytical form. This model is illustrated by simulations and experiments in Section 3. In Section 4, after recalling the fractional Fourier transformation (FRFT) operator, we show that hologram reconstruction can be achieved by FRFT.

\section{DIGITAL IN-LINE HOLOGRAPHY WITH A SPATIALLY PARTIALLY COHERENT SOURCE}

Spatially partially coherent sources are already widely used in digital holography. Nevertheless, the analytical theory of the distribution of the intensity of the spatially partially coherent field diffracted by a particle is not sufficiently developed. The advantage of the knowledge of the recorded intensity is that one can study the influence of the different parameters of the experimental set-up and the effects of the spatially partially coherent beam on recorded holograms can be predicted. Figure 1 represents the numerical and experimental setup in which all parameters are identified.

To obtain a spatially partially coherent source, it is possible to 


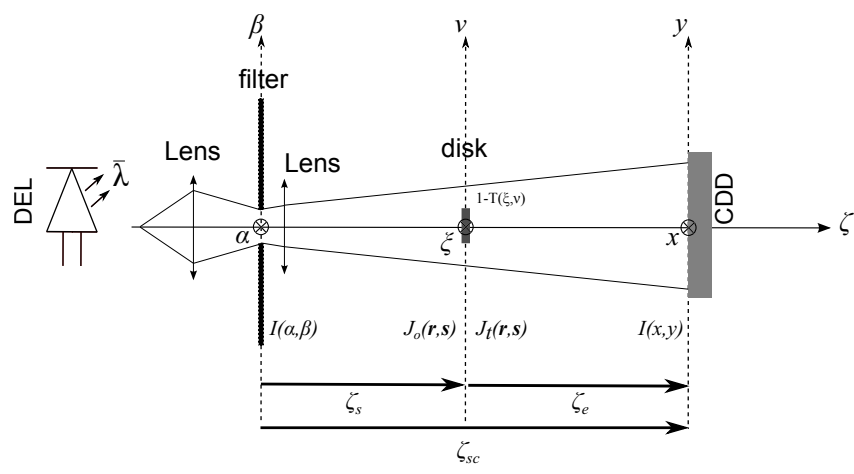

FIG. 1 Experimental set-up for digital in-line holography from propagation of the mutual intensity function. $\zeta_{s}$ : distance between the filter and the particle, $\zeta_{e}$ : the distance between the particle and the CCD sensor.

use a coherent spatial source [16]. Alternatively, it is possible to use a LED. The LED (light-emitting diode) can be a red with mean wavelength $\lambda_{m}=625 \mathrm{~nm}$ or blue with $\lambda_{m}=455 \mathrm{~nm}$. The used LED at $\lambda_{m}=625 \mathrm{~nm}$ has an experimental bandwidth in $21.5 \mathrm{~nm}$ range. This would have a small effect in the results since one should then add contributions at different wavelengths incoherently. The resulting scaling range in the diffraction pattern is small. Moreover, . Note that here, These are the interactions between the source and the object of interest and not the intrinsic characteristics of the source even if we know it is related. The first lens focuses the beam over the pinhole filter. It is considered that the output numerical aperture of the first lens before the pinhole filter is very large. The effective coherence area is much smaller than the area of the filter. Then, the assumption of spatially partially coherent illumination is valid in practise. In the $(\alpha, \beta)$-plane, the pinhole filter is placed with $A$ and $B$ the respective apertures along the axes. The opaque disk of diameter $D_{t h}$ is localized at distance $\zeta_{s}$ from the pinhole and at distance $\zeta_{e}$ from the CCD sensor. The distance between the filter and the CCD sensor is denoted by $\zeta_{s c}$. In the spatial coherence point of view, the cross-correlation between two spatial points from the wavefront is needed. For this, two points along each axis must be considered. Along the $\xi$-axis the two points are $\xi_{1}$ and $\xi_{2}$. Along the $v$-axis, the two points are $v_{1}$ and $v_{2}$. By noting that $\Delta \xi=\xi_{2}-\xi_{1}, \Delta v=v_{2}-v_{1}$, $\xi=\left(\xi_{2}+\xi_{1}\right) / 2, v=\left(v_{2}+v_{1}\right) / 2$ and from Eq. (5.6-8) on p. 209 of [17], the mutual intensity function $\widehat{J}_{o}(\xi, \Delta \xi, v, \Delta v)$ in the plane just before the opaque disk versus the intensity distribution of the Gaussian filter is expressed by means of Van Cittert-Zernike theorem according to

$$
\begin{array}{r}
\widehat{J}_{o}(\xi, \Delta \xi, v, \Delta v)=\frac{\lambda_{m}^{2}}{\pi\left(\lambda_{m} \zeta_{s}\right)^{2}} \exp \left[-i 2 \pi \frac{\xi \Delta \xi+v \Delta v}{\lambda_{m} \zeta_{s}}\right] \\
\times \int_{\mathbb{R}^{2}} I(\alpha, \beta) \cdot \exp \left[i \frac{2 \pi}{\lambda_{m} \zeta_{s}}(\beta \Delta \xi+\alpha \Delta v)\right] d \alpha d \beta,
\end{array}
$$

where $\widehat{J}_{o}(\xi, \Delta \xi, v, \Delta v)=J_{o}(\xi-\Delta \xi / 2, v-\Delta v / 2, \xi+\Delta \xi / 2, v+$ $\Delta v / 2)$ and the Gaussian aperture filter $I(\alpha, \beta)$ is given by, see [18], Eq. (29) on p.786

$$
I(\alpha, \beta)=I_{0} \exp \left[-\left(\frac{\alpha^{2}}{A^{2}}+\frac{\beta^{2}}{B^{2}}\right)\right],
$$

with $I_{0}=I(0,0)$ and $A, B$ the $1 / e^{2}$ width of the filter. It is important to note that we have considered the general case, i.e. $A \neq B$, in the theoretical developments but from the practical experiences we have chosen $A \approx B$. These cases correspond to an elliptical and a circular filter, respectively. The combination of Eqs. (2) and (1) gives us the following mutual intensity function $\widehat{J}_{o}$ :

$$
\begin{aligned}
& \widehat{J}_{o}(\xi, \Delta \xi, v, \Delta v)=K \exp \left(-\frac{\pi^{2} A^{2}}{\left(\lambda_{m} \zeta_{s}\right)^{2}} \Delta \xi^{2}\right) \\
& \quad \times \exp \left(-\frac{\pi^{2} B^{2}}{\left(\lambda_{m} \zeta_{s}\right)^{2}} \Delta v^{2}\right) \times \exp \left[-i 2 \pi \frac{\xi \Delta \xi+v \Delta v}{\lambda_{m} \zeta_{s}}\right]
\end{aligned}
$$

where $K=\frac{\lambda_{m}^{2} A B I_{0}}{\left(\lambda_{m} \zeta_{s}\right)^{2}}$. Note that the mutual intensity function is a Gaussian function involving $\xi_{1}, \xi_{2}, v_{1}, v_{2}$. This situation is reminiscent of the situation in [12] where an incident elliptic and astigmatic Gaussian beam illuminates an opaque disk. In [12], the high frequencies in the diffraction pattern are smoothed in a more regular way than what one gets with a circular filter where the mutual intensity function is governed by a Bessel function. The spatial transmittance of the opaque 2D-opaque disk is defined by $[1-T(\xi, v)]$. From [17], Eq. (5.74) on p.223, the mutual intensity function, denoted by $\widehat{J}_{t}$, of the transmitted light is thus

$$
\begin{aligned}
\widehat{J}_{t}(\xi, \Delta \xi, v, \Delta v) & =\left[1-T\left(\xi-\frac{\Delta \xi}{2}, v-\frac{\Delta v}{2}\right)\right] \\
\times & {\left[1-\bar{T}\left(\xi+\frac{\Delta \xi}{2}, v+\frac{\Delta v}{2}\right)\right] \times \widehat{J}_{o}(\xi, \Delta \xi, v, \Delta v) . }
\end{aligned}
$$

The overhead bar on $T$ denotes the complex conjugation of $T$. As $T$ is real then $\bar{T}=T$. To calculate the observed intensity distribution of the interferences between the reference beam and the part of this beam diffracted by the opaque disk, we begin with [17], Eq. (5.7-6) on p.224 in the paraxial approximation. Then, the intensity is given by:

$$
\begin{aligned}
I(x, y)= & \frac{1}{\left(\lambda_{m} \zeta_{e}\right)^{2}} \int_{\mathbb{R}^{4}} \widehat{J}_{t}(\xi, \Delta \xi, v, \Delta v) \\
& \times \exp \left[-i \frac{2 \pi}{\lambda_{m} \zeta_{e}}(\xi \Delta \xi+v \Delta v)\right] \\
& \times \exp \left[i \frac{2 \pi}{\lambda_{m} \zeta_{e}}(x \Delta \xi+y \Delta v)\right] d \xi d v d \Delta \xi d \Delta v
\end{aligned}
$$

In the plane of the CCD sensor, the intensity distribution $I(x, y)$ is also the sum of four terms, i.e.:

$$
I(x, y)=I_{1}-\left[I_{2}+I_{3}\right]+I_{4} .
$$

These four terms arise from expanding of Eq. (4) into four terms and expanding Eq. (5) accordingly.

\subsection{Expression for $I_{1}(x, y)$}

The first term $I_{1}$ at the right-hand side of Eq. (6) is given by

$$
\begin{aligned}
I_{1}(x, y)= & \frac{1}{\left(\lambda_{m} \zeta_{e}\right)^{2}} \int_{\mathbb{R}^{4}} \widehat{J}_{o}(\xi, \Delta \xi, v, \Delta v) \\
& \times \exp \left[-i \frac{2 \pi}{\lambda_{m} \zeta_{e}}(\xi \Delta \xi+v \Delta v)\right] \\
& \times \exp \left[i \frac{2 \pi}{\lambda_{m} \zeta_{e}}(x \Delta \xi+y \Delta v)\right] d \xi d v d \Delta \xi d \Delta v
\end{aligned}
$$

This $I_{1}$ is expressed in 7.1 of appendix 7 as

$$
I_{1}(x, y)=\frac{A B}{\left(\zeta_{s}+\zeta_{e}\right)^{2}} \cdot I_{0} .
$$


This Eq. (41) embodies the transition to the spatially partially coherent case from the coherent case in Eq. (25).

\subsection{Expression for $I_{2}(x, y)$ and $I_{3}(x, y)$}

Now, for the second term $I_{2}$, we have to calculate the following integral:

$$
\begin{aligned}
I_{2}(x, y)= & \frac{1}{\left(\lambda_{m} \zeta_{e}\right)^{2}} \int_{\mathbb{R}^{4}} T\left(\xi-\frac{\Delta \xi}{2}, v-\frac{\Delta v}{2}\right) \widehat{J}_{o}(\xi, \Delta \xi, v, \Delta v) \\
& \times \exp \left[-i \frac{2 \pi}{\lambda_{m} \zeta_{e}}(\xi \Delta \xi+v \Delta v)\right] \\
& \times \exp \left[i \frac{2 \pi}{\lambda_{m} \zeta_{e}}(x \Delta \xi+y \Delta v)\right] d \xi d v d \Delta \xi d \Delta v .
\end{aligned}
$$

In 7.2 of appendix 7 , this $I_{2}$ is expressed as

$$
\begin{aligned}
I_{2}(r, \varphi)= & \frac{\pi^{2} K D_{t h}^{2}}{\left(\lambda \zeta_{e}\right)^{2} \sqrt{M N}} \cdot \exp \left[-\left(\alpha_{x} x^{2}+\alpha_{y} y^{2}\right)\right] \\
& \cdot \sum_{k=0}^{\infty}(-i)^{k} \varepsilon_{k} T_{k}(r, \gamma) \cos (2 k \varphi) .
\end{aligned}
$$

The exponential function at the right-hand side of Eq. (10) is a linearly chirped Gaussian controlled by the parameters $\alpha_{x}$ and $\alpha_{y}$ defined by

$$
\alpha_{x}=\frac{\pi^{2}}{\left(\lambda_{m} \zeta_{e}\right)^{2}} \frac{\bar{M}}{|M|^{2}}, \quad \alpha_{y}=\frac{\pi^{2}}{\left(\lambda_{m} \zeta_{e}\right)^{2}} \frac{\bar{N}}{|N|^{2}}
$$

with

$$
\begin{gathered}
M=\frac{\pi^{2} A^{2}}{\left(\lambda_{m} \zeta_{s}\right)^{2}}+i \pi L, \quad N=\frac{\pi^{2} B^{2}}{\left(\lambda_{m} \zeta_{s}\right)^{2}}+i \pi L, \\
L=\left(\frac{1}{\lambda_{m} \zeta_{e}}+\frac{1}{\lambda_{m} \zeta_{s}}\right),
\end{gathered}
$$

Furthermore, $r$ and $\varphi$ are such that

$$
r \cos \varphi=a, \quad r \sin \varphi=b,
$$

with

$$
a=-i L \frac{\pi^{2} D_{t h}}{\lambda_{m} \zeta_{e}} \cdot \frac{\bar{M}}{|M|^{2}} \cdot x, \quad b=-i L \frac{\pi^{2} D_{t h}}{\lambda_{m} \zeta_{e}} \cdot \frac{\bar{N}}{|N|^{2}} \cdot y,
$$

by assuming that $a \pm i b \neq 0$, see appendix 8 , which is generally the case from experimental point of view. The parameter $\gamma$ is given by

$$
\gamma=i \pi^{2} \frac{D_{t h}^{2}}{8}\left(\frac{\bar{M}}{|M|^{2}}+\frac{\bar{N}}{|N|^{2}}\right) L^{2},
$$

and it is linked to the apertures of the filter and to the position of the opaque disk along the optical-axis from the CCD sensor and from the filter. The functions $T_{k}$ (see Eqs. (51) and (18)) are given in terms of Bessel functions as follows:

$$
T_{k}(r, \gamma)=\sum_{p=0}^{\infty} \beta_{2 k+2 p}^{2 k}(\delta) V_{2 k+2 p}^{2 k}(r, \gamma)
$$

The parameters $\delta$ is given by

$$
\delta=i \pi^{2} \frac{D_{t h}^{2}}{8}\left(\frac{\bar{M}}{|M|^{2}}-\frac{\bar{N}}{|N|^{2}}\right) L^{2}
$$

The $\delta$-parameter is linked to the ellipticity of the filter. Indeed, in the particular case where the filter is circular, we have $\delta=0$. In 7.2 in appendix 7 , the expansion coefficients $\beta_{2 k+2 p}^{2 k}$ are expressed explicitly in terms of the hypergeometric functions ${ }_{2} F_{3}$ as in [12]. Note that, in the case of an elliptical filter $\beta_{2 k+2 p}^{2 k}(0)=1$ if $k=p=0$ and 0 otherwise. The V-functions have the series expression $([12,29,28])$ :

$$
V_{n}^{m}(r, f)=\exp (i f) \cdot \sum_{l=1}^{\infty}(-2 i f)^{l-1} \sum_{j=0}^{P} v_{l j} \cdot \frac{J_{m+l+2 j}(r)}{l(r)^{l}},
$$

where $n$ and $m$ are integers $\geq 0$ with $n-m$ even and nonnegative, and

$$
\begin{aligned}
v_{l j}= & (-1)^{P}(m+l+2 j)\left(\begin{array}{c}
m+j+l-1 \\
l-1
\end{array}\right)\left(\begin{array}{c}
j+l-1 \\
l-1
\end{array}\right) \\
& \times\left(\begin{array}{c}
l-1 \\
P-j
\end{array}\right) /\left(\begin{array}{c}
Q+l+j \\
l
\end{array}\right)
\end{aligned}
$$

for $l=1,2, \ldots, j=0,1, \ldots, P, P=\frac{n-m}{2}$ and $Q=\frac{n+m}{2}$. Furthermore, $I_{3}$ is the complex conjugate of $I_{2}$. Consequently, the sum of $I_{2}$ and $I_{3}$ gives us the following result:

$$
I_{2}(r, \varphi)+I_{3}(r, \varphi)=I_{2}(r, \varphi)+\overline{I_{2}(r, \varphi)}=2 \cdot \Re\left\{I_{2}(r, \varphi)\right\},
$$

where $\Re$ denotes the real part. Eq. (20) can be compared to the second term at the right-hand side of Eq. (25) where the linearly chirped Gaussian is replaced by the real linear chirp of Eq. (10) and the V-functions defined in Eq. (18) replace the Jinc $_{1}$-function in Eq. (25). One thus see that the mathematical structure is the same in the case of a spatially partially coherent source.

\subsection{Expression for $I_{4}$}

To derive a semi-analytic expression for

$$
\begin{aligned}
I_{4}(x, y)= & \frac{1}{\left(\lambda_{m} \zeta_{e}\right)^{2}} \int_{\mathbb{R}^{4}} T\left(\xi-\frac{\Delta \xi}{2}, v-\frac{\Delta v}{2}\right) \\
& \times T\left(\xi+\frac{\Delta \xi}{2}, v+\frac{\Delta v}{2}\right) \widehat{J}_{o}(\xi, \Delta \xi, v, \Delta v) \\
& \times \exp \left[-i \frac{2 \pi}{\lambda_{m} \zeta_{e}}(\xi \Delta \xi+v \Delta v)\right] \\
& \times \exp \left[i \frac{2 \pi}{\lambda_{m} \zeta_{e}}(x \Delta \xi+y \Delta v)\right] d \xi d v d \Delta \xi d \Delta v,
\end{aligned}
$$

it is necessary to use mathematical results on (i) a special Zernike expansion, (ii) the correlation product of Zernike polynomials [30], (iii) the linearization of products of Zernike polynomials [31], and (iv), the extended Nijboer-Zernike theory (ENZ)[29]. The details of the computations are given in 7.3 of appendix 7. The semi-analytical expression for the integral $I_{4}$ is then

$$
\begin{aligned}
I_{4}(r, \varphi)= & \frac{\pi^{2} D_{t h}^{4}}{8} \frac{K}{\left(\lambda_{m} \zeta_{e}\right)^{2}} \sum_{q=0}^{\infty} C_{q}(\kappa) \sum_{n=-\infty}^{\infty} \sum_{p=0}^{\infty} i^{n} \beta_{|2 n|+2 p}^{|2 n|}(\psi) \\
& \times \sum_{s=\max (0, p-q, q-p-|2 n|)}^{p+q} A_{|2 n|+2 p, 2 q,|2 n|+2 s}^{|2 n|, 0,2 n \mid} \\
& \times V_{|2 n|+2 s}^{|2 n|}(2 r, \chi) \exp [i 2 n \varphi]
\end{aligned}
$$


with

$$
C_{q}(\kappa)=\sum_{l, l^{\prime}}^{\infty} \frac{(2 q+1) \Gamma_{2 l, 2 l^{\prime}, 2 q}^{000}}{(2 l+1)\left(2 l^{\prime}+1\right)} \mathcal{B}_{2 l}^{0}(\kappa) \overline{\mathcal{B}_{2 l^{\prime}}^{0}(\kappa)},
$$

where the $\Gamma_{2 l, 2 l^{\prime}, 2 q}^{000}$ are expressed in terms of at most four Jacobi polynomials evaluated at 0 , the $\mathcal{B}_{2 l}^{0}$ are expansion coefficients involving spherical Bessel functions of the first kind, $\beta|2 n|+2 p$ are expressed in terms of the hypergeometric function ${ }_{2} F_{3}$, the $A_{|2 n|+2 n, 2 q,|2 n|+2 s}^{|2 n|, 0,|2 n|}$ are Wigner coefficients, and $V_{|2 n|+2 s}^{|2 n|}$ are functions from ENZ theory as in Eq. (18). The coefficients $\kappa, \psi, \chi, \mathcal{A}$ and $\mathcal{B}$ are as follows:

$$
\begin{gathered}
\mathcal{\kappa}=\frac{L D_{t h}^{2}}{4}, \quad \psi=i 2(\mathcal{A}-\mathcal{B}) \quad \chi=2 i(\mathcal{A}+\mathcal{B}), \\
\mathcal{A}=\frac{\pi^{2} D_{t h}^{2}}{4} \frac{A^{2}}{\left(\lambda \zeta_{s}\right)^{2}}, \quad \mathcal{B}=\frac{\pi^{2} D_{t h}^{2}}{4} \frac{B^{2}}{\left(\lambda \zeta_{s}\right)^{2}} .
\end{gathered}
$$

As one can see, this fourth term, $I_{4}$, is expressed in terms of Bessel functions, (just as the last terms of Eq. (25)). The only difference is that the square of the Jinc ${ }_{1}$-function is replaced by a series involving V-functions.

\subsection{Particular case: $\mathcal{A}=\mathcal{B}=0$}

In this particular case, the mathematical model of the intensity distribution obtained with partially spatially coherent beam must tend to the intensity distribution obtained with a laser. In the case of an opaque disk, the mathematical definition of the normalized intensity distribution in far-field approximation (i.e., $\pi D_{t h}^{2} / 2 \lambda z \ll 1$ in [12], Eq. (18)), denoted in Eq. (25) as $I_{c o h}(r)$, is

$$
\begin{aligned}
I_{c o h}(r)=1 & -\frac{\pi D_{t h}^{2}}{\lambda z} \sin \left[\frac{\pi r^{2}}{\lambda z}\right] \cdot \frac{J_{1}\left(\frac{\pi D_{t h} r}{\lambda z}\right)}{\frac{\pi D_{t h} r}{\lambda z}} \\
& +\left[\frac{\pi D_{t h}^{2}}{2 \lambda z} \frac{J_{1}\left(\frac{\pi D_{t h} r}{\lambda z}\right)}{\frac{\pi D_{t h} r}{\lambda z}}\right]^{2}
\end{aligned}
$$

To treat this case, Eq. (6) must be normalized by the coefficient $K=\frac{\lambda_{m}^{2} A B I_{0}}{\left(\lambda_{m} \zeta_{s}\right)^{2}}$. Then, in the far-field approximation, i.e. $\zeta_{s} \gg \zeta_{e}$,

$$
\lim _{\mathcal{A} \rightarrow 0} \frac{I_{1}}{K}=1
$$

This limit corresponds to the first term of the Eq. (25). In the same way, the second and third terms,

$$
\lim _{\mathcal{A} \rightarrow 0} \frac{I_{2}+I_{3}}{K}=\frac{\pi D_{t h}^{2}}{\lambda_{m} \zeta_{e}} \frac{J_{1}\left(\frac{\pi D_{t h} r}{\lambda z}\right)}{\frac{\pi D_{t h} r}{\lambda z}}
$$

And for the fourth term, we have, by identification:

$$
\begin{aligned}
& \lim _{\mathcal{A} \rightarrow 0} \frac{I_{4}}{K}=\frac{\pi^{2} D_{t h}^{4}}{8\left(\lambda_{m} \zeta_{e}\right)^{2}} \cdot \sum_{q=0}^{\infty} C_{q}(\kappa) \cdot V_{2 q}^{0}(4 \pi r, 0) \\
= & {\left[\frac{\pi D_{t h}^{2}}{2 \lambda z} \frac{J_{1}\left(\frac{\pi D_{t h} r}{\lambda z}\right)}{\frac{\pi D_{t h} r}{\lambda z}}\right]^{2}, }
\end{aligned}
$$

with $r=\frac{D_{t h}}{2 \lambda_{m} \zeta_{e}}\left(x^{2}+y^{2}\right)^{1 / 2}$.

\section{NUMERICAL EXPERIMENT AND EXPERIMENTALRESULT}

Firstly, in the theoretical developments, the filter is considered as a Gaussian function (see Eq. (2)) to simplify the calculus. In the experiences, the filter is a pinhole. As we will see it, the results are similar. However, it is possible to consider the method of Gaussian functions superposition to describe the pinhole $[13,14]$. Secondly, knowing that the theoretical curvature of the wave in the plane of the object is defined by the quadratic phase of Eq. (3), the second lens has two purposes: it allows to collect the maximum energy focused by the first lens, and adjusts the experimental curvature of the wave with the theoretical curvature of (3). In practical experiments, one has typically $D_{t h} \approx 10^{-4} \mathrm{~m}, \zeta_{e, s} \approx 10^{-2} \mathrm{~m}$ and $\lambda \approx 10^{-6} \mathrm{~m}$. Then $\kappa \approx 0.5$. The apertures $A$ and $B$ of the pinhole are approximately of the order of $10^{-4} \mathrm{~m}$. Then $|\psi| \approx 3.70$ and $|\chi| \approx 10$. If the pinhole is close to being circular, $A \approx B$ then $|\psi| \approx 0^{+}$. Furthermore, $\delta \approx-0.454+0.0885 i$ and $\gamma \approx 0.907+0.623 i$. As the diameter $D_{t h}$ is of the order of or less than $10^{-4} \mathrm{~m}$ we have that $\kappa, \psi, \delta, \gamma$ and $\chi$ are of the order of or less than $10^{-2}$. With these values, we can limit the truncation of the sums at 3 in Eq. (23), at $l=5$ in Eq. (18), at $p=5$ in Eq. (51), and at $k=3$ in Eq. (10). In order to give an illustration of our results, we first have simulated diffraction patterns produced by an opaque disk of $80 \mu \mathrm{m}$ diameter. The aperture values of the pinhole are $A=50 \mu \mathrm{m}$ and $B=50.1 \mu \mathrm{m}$. The particle is localized at $\zeta_{s}=216 \mathrm{~mm}$ from the pinhole and at $\zeta_{e}=80 \mathrm{~mm}$ from the CCD sensor. Figure 2(a) shows the simulated intensity distribution at the observation plane and Figure 2(b) the experimental intensity distribution recorded by the CCD sensor.

The holograms consist of a $1000 \times 1000$ array of $4.4 \mu \mathrm{m} \times 4.4 \mu \mathrm{m}$ size pixels. The mean wavelength of the LED source is equal to $\lambda_{m}=625 \mathrm{~nm}$. These illustrations reveal a good agreement between numerical and experimental diffraction patterns with a partially coherent source. To confirm this point, the transverse intensity profiles obtained from Figure 2(a) and Figure 2(b) are presented in Figure 4.

Here the intensity distribution has been modified so as to match the experimental grey level of each pixel with the theoretical values.

In this first result the effective coherence area of the beam is equal to $1.158 \mathrm{~mm}^{2}$ while the object's area is equal to $0.005 \mathrm{~mm}^{2}$. This situation is closed to an object illuminated by a coherence beam. However, the choice of apertures $A$ and $B$ must allow us then to demonstrate the capability to reconstruct the image of the object and to deduce the axial position $\zeta_{e}$ and to estimate its diameter $D_{t h}$. If the effective coherence is equal to the object's area, the aperture of the filter must be equal to $\mathrm{A}=\mathrm{B}=759.6 \mu \mathrm{m}$. With this aperture, it is not possible to reconstruct the image of the particle. Thus, a metrology of the reconstructed image is not possible. Note that again, when the apertures $A, B$ are greater than $200 \mu \mathrm{m}$, the diffraction pattern does not contained interference rings. At these apertures, we consider that a metrology on the reconstructed image of the object is not possible. 


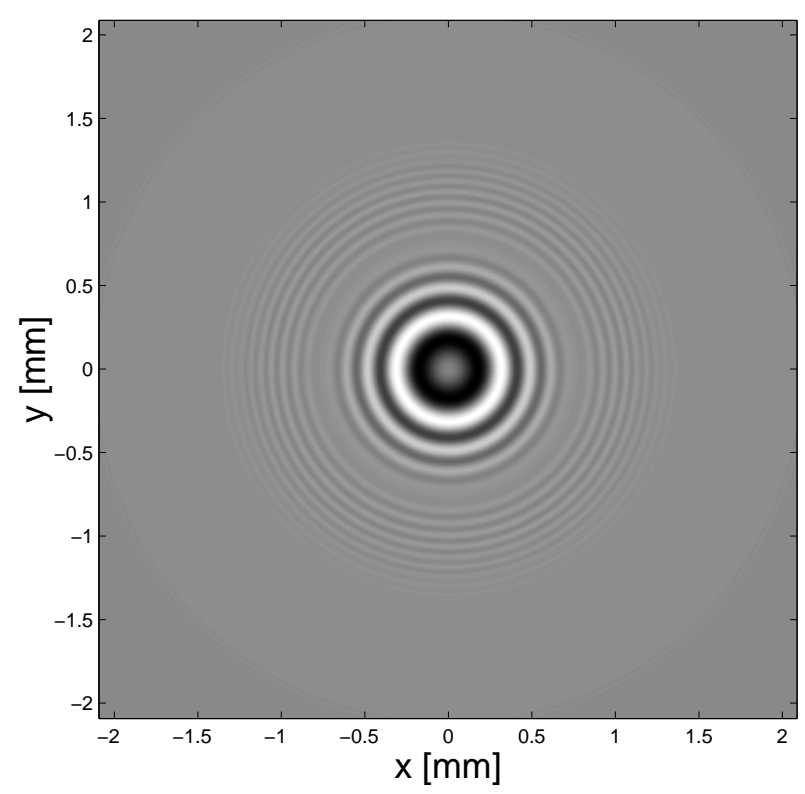

FIG. 2 Theoretical result of the intensity distributions for $A=50 \mu \mathrm{m}, B=50.1 \mu \mathrm{m}$, $\zeta_{s}=216 \mathrm{~mm}, D_{t h}=80 \mu \mathrm{m}, \zeta_{e}=80 \mathrm{~mm}$.

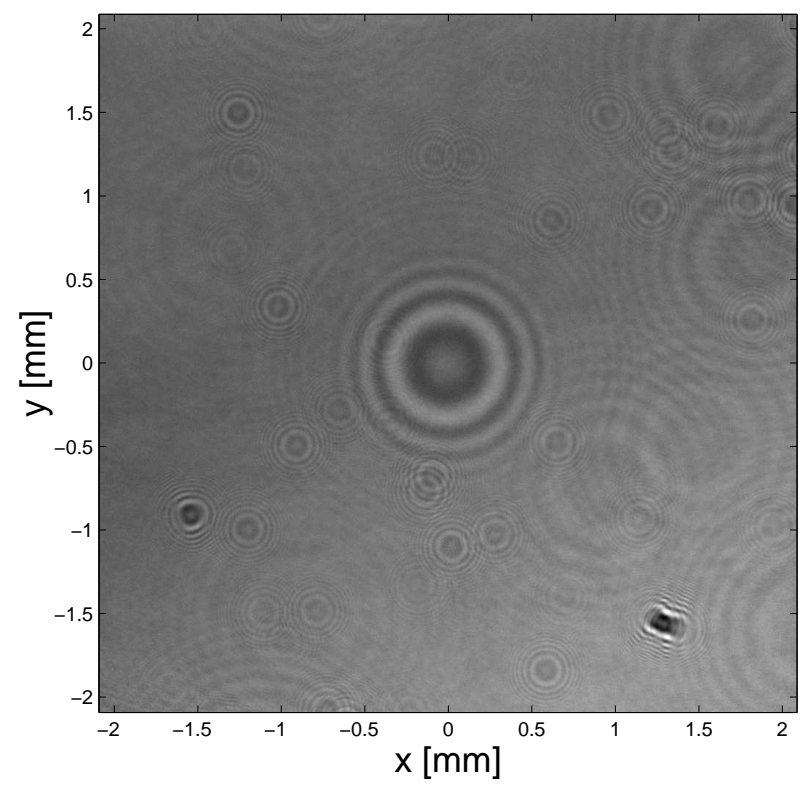

FIC. 3 Experimental result of the intensity distributions for $A=50 \mu \mathrm{m}, B=50.1 \mu \mathrm{m}$, $\zeta_{s}=216 \mathrm{~mm}, D_{t h}=80 \mu \mathrm{m}, \zeta_{e}=80 \mathrm{~mm}$.

Now, as the theoretical developments are in accordance with the experimental result, remember that the purpose is to obtain an image of the opaque disk by means of a digital refocusing. This is precisely the role of the fractional Fourier transformation, and this is elaborated in the next section.

\section{DIGITAL IN-LINE RECONSTRUCTION BY FRACTIONAL FOURIER TRANSFORMATION ANALYSIS}

Fractional Fourier Transformation (FRFT) is an integral operator that has various application in signal and image processing. Its mathematical definition is given in Ref. [25, 24, 26]. The two-dimensional fractional Fourier transformation of order $a_{x}$ for $x$-cross-section and $a_{y}$ for $y$-cross-section with

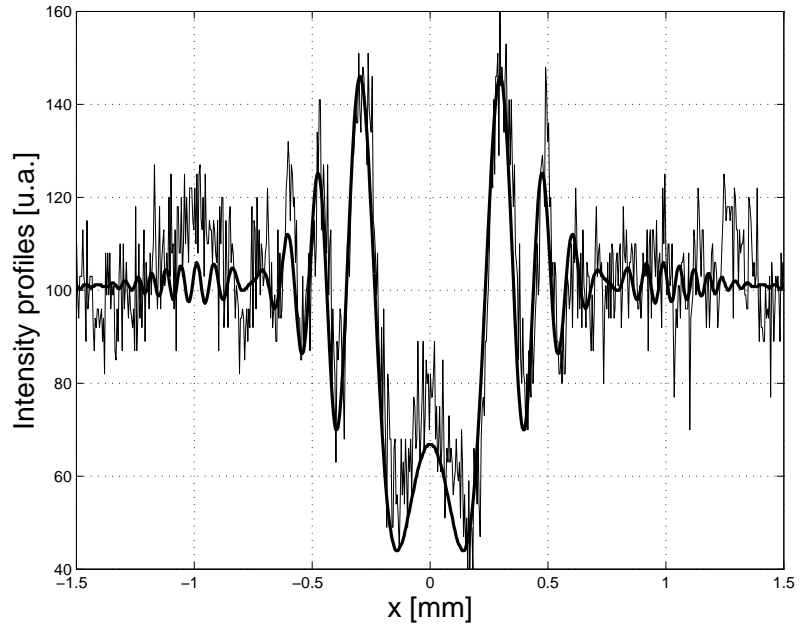

FIG. 4 Comparison between simulated and experimental intensity distributions: theoretical result (solid width line), experimental result (solid line).

$0 \leq\left|\theta_{x}\right| \leq \pi / 2$ and $0 \leq\left|\theta_{y}\right| \leq \pi / 2$, respectively, of a 2Dfunction $I(x, y)$ is defined as (with $\theta_{j}=\frac{a_{j} \pi}{2}$ and $j=x, y$ )

$$
\begin{aligned}
& \mathcal{F}_{\theta_{x}, \theta_{y}}[I(x, y)]\left(x_{a}, y_{a}\right) \\
= & \int_{\mathbb{R}^{2}} N_{\theta_{x}}\left(x, x_{a}\right) N_{\theta_{y}}\left(y, y_{a}\right) I(x, y) d x d y,
\end{aligned}
$$

where the kernel of the fractional operator is defined by

$$
N_{\theta_{j}}\left(x, x_{a}\right)=C\left(\theta_{j}\right) \exp \left(i \pi \frac{x^{2}+x_{a}^{2}}{s_{j}^{2} \tan \theta_{j}}\right) \exp \left(-\frac{i 2 \pi x_{a} x}{s_{j}^{2} \sin \theta_{j}}\right) \text {, }
$$

and

$$
C\left(\theta_{j}\right)=\frac{\exp \left(-i\left(\frac{\pi}{4} \operatorname{sign}\left(\sin \theta_{j}\right)-\frac{\theta_{j}}{2}\right)\right)}{\left|s_{j}^{2} \sin \theta_{j}\right|^{1 / 2}}
$$

Generally, the parameter $s_{j}$ is considered as a normalization constant. It can take any value. In our case, its value is defined from the experimental set-up according to [20]: $s_{j}^{2}=N_{j} \cdot \delta_{j}^{2}$. Recall that the aim is to reconstruct the image of the object. To do this, we know from literature $[9,21,15,22,23]$ that the second or the third term of Eq. (6), containing the linear chirp, allows us a digital refocusing to form images from recorded holograms. We write the fractional Fourier transformation of $I$ in Eq. (6) as

$$
\begin{aligned}
\mathcal{F}_{\theta_{x}, \theta_{y}}[I]\left(x_{a}, y_{a}\right)= & \mathcal{F}_{\theta_{x}, \theta_{y}}\left[I_{1}\right]-\mathcal{F}_{\theta_{x}, \theta_{y}}\left[I_{2}\right]-\mathcal{F}_{\theta_{x}, \theta_{y}}\left[I_{3}\right] \\
& +\mathcal{F}_{\theta_{x}, \theta_{y}}\left[I_{4}\right] .
\end{aligned}
$$

As to the second or third term of Eq. (31), the basic assumption is that the linear chirps of the FRFT, controlled by the fractional orders, cancel the linear chirps contained in $I_{2}$ or $I_{3}$. Consequently, for the term $\mathcal{F}_{\theta_{x}, \theta_{y}}\left[I_{2}\right]$, the following conditions must be satisfied:

$$
\cot \theta_{x o}=-\frac{s_{x}^{2}}{\pi} \cdot \Im\left\{\alpha_{x}\right\} \text { and } \cot \theta_{y o}=-\frac{s_{y}^{2}}{\pi} \cdot \Im\left\{\alpha_{y}\right\} .
$$

Here $\Im\{$.$\} denotes the imaginary part. The coefficients \theta_{x o, y o}$ are the optimal fractional orders to reconstruct the image of the particle. For example, the reconstructed image of the $80 \mu \mathrm{m}$ diameter particle from the diffraction pattern in Figure 2(a) with $a_{x}=a_{y}=0.833091$ is illustrated in Figure 5. 


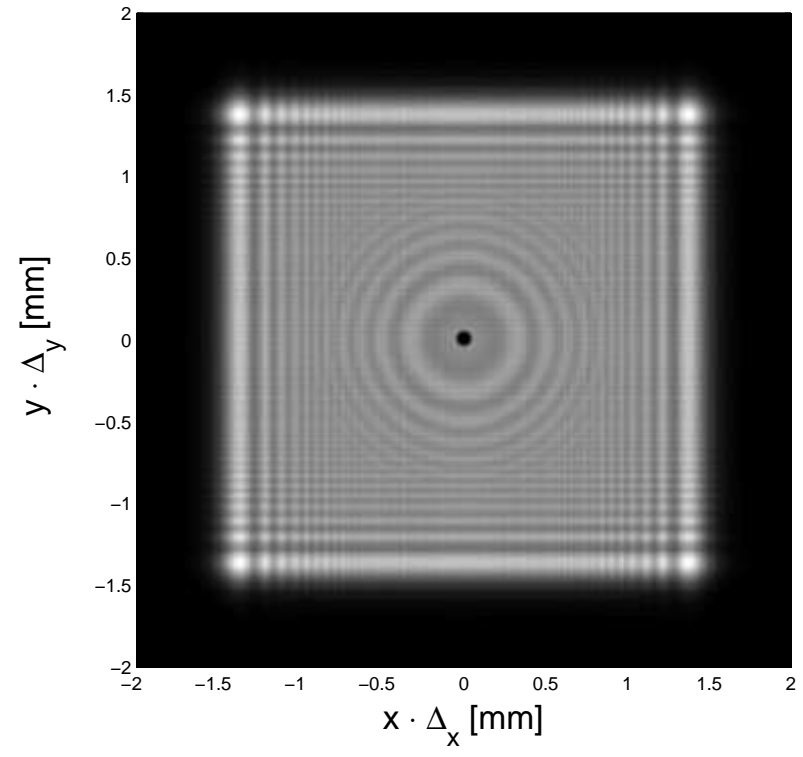

FIG. 5 Reconstruction of the image of the particle with the FRFT: $a_{x}=a_{y}=0.833091$, $N_{x}=N_{y}=950$

Conversely, the knowledge of these optimized fractional orders leads to the determination of the position $\zeta_{e}$ of the opaque disk from one of the two Eqs. (32) as the real root of the cubic equation

$$
\zeta_{e}^{3}-a_{1} \cdot \zeta_{e}^{2}+a_{2} \cdot \zeta_{e}-a_{3}=0
$$

with

$$
\begin{aligned}
a_{1} & =\frac{2 \lambda_{m}^{2} \cdot \zeta_{s c}^{3} \cot \theta_{x o}+3 \lambda_{m} \cdot s_{x}^{2} \cdot \zeta_{s c}^{2}}{\pi^{2} A^{4} \cot \theta_{x o}+\lambda_{m}^{2} \zeta_{s c}^{2} \cot \theta_{x o}+\lambda s_{x}^{2} \zeta_{s c}} \\
a_{2} & =\frac{\lambda_{m}^{2} \cdot \zeta_{s c}^{4} \cot \theta_{x o}+3 \lambda_{m} \cdot s_{x}^{2} \cdot \zeta_{s c}^{3}}{\pi^{2} A^{4} \cot \theta_{x o}+\lambda_{m}^{2} \zeta_{s c}^{2} \cot \theta_{x o}+\lambda s_{x}^{2} \zeta_{s c}} \\
a_{3}= & \frac{\lambda_{m} \cdot s_{x}^{2} \cdot \zeta_{s c}^{4}}{\pi^{2} A^{4} \cot \theta_{x o}+\lambda_{m}^{2} \cdot \zeta_{s c}^{2} \cot \theta_{x o}+\lambda s_{x}^{2} \zeta_{s c}}
\end{aligned}
$$

If Eqs. (32) are satisfied, to evaluate the second term in Eq. (31), an approximation of $I_{2}$ for $k=p=0$ and $l=1$ must be done and gives

$$
\begin{aligned}
I_{2}= & \frac{\pi^{2} K D_{t h}^{2}}{\left(\lambda \zeta_{e}\right)^{2} \sqrt{M N}} \exp \left[-\left(\alpha_{x r} \cdot x^{2}+\alpha_{y r} \cdot y^{2}\right)\right] \\
& \times \frac{1}{2} \beta_{0}^{0}(\delta) \exp (i \gamma) \frac{J_{1}(r)}{r}
\end{aligned}
$$

The optimal fractional Fourier transformation of $I_{2}$ is then

$$
\begin{aligned}
& \mathcal{F}_{\theta_{x o}, \theta_{y o}}\left[I_{2}\right]\left(x_{a}, y_{a}\right)=\mathcal{C}\left(x_{a}, y_{a}\right) \\
& \cdot \int_{\mathbb{R}^{2}} \exp \left[-\left(\alpha_{x r} \cdot x^{2}+\alpha_{y r} \cdot y^{2}\right)\right] \frac{J_{1}(r)}{r} \\
& \cdot \exp \left[-i 2 \pi\left(\frac{x_{a} x}{s_{x}^{2} \sin \theta_{x o}}+\frac{y_{a} y}{s_{y}^{2} \sin \theta_{y o}}\right)\right] d x d y,
\end{aligned}
$$

with

$$
\begin{aligned}
\mathcal{C}\left(x_{a}, y_{a}\right)= & C\left(\theta_{x o}\right) C\left(\theta_{y o}\right) \frac{\pi^{2} K D^{2}}{2\left(\lambda_{m} \zeta_{e}\right)^{2}} \frac{\beta_{0}^{0}(\delta)}{(M N)^{1 / 2}} \\
& \times \exp (i \gamma) \exp \left[i \pi\left(x_{a}^{2} \cot \theta_{x o}+y_{a}^{2} \cot \theta_{y o}\right)\right] .
\end{aligned}
$$

The function $\mathcal{C}\left(x_{a}, y_{a}\right)$ only appears as a proportionality constant because we work in intensity. The Eq. (36) is no more than a classical 2D Fourier transformation over the frequencies $(u, v)$ of the product of two functions which is the convolution of their transforms, thus

$$
\begin{aligned}
& \mathcal{F} I_{2}(u, v)=\mathcal{C}\left(u \cdot s_{x}^{2} \sin \theta_{x o}, v \cdot s_{y}^{2} \sin \theta_{y o}\right) \\
& \quad \times\left(\mathcal{F}\left[\exp \left[-\left(\alpha_{x r} \cdot x^{2}+\alpha_{y r} \cdot y^{2}\right)\right]\right]_{\text {conv }}^{* *} \mathcal{F}\left[\frac{J_{1}(r)}{r}\right]\right),
\end{aligned}
$$

with the coordinates $x_{a}=u \cdot s_{x}^{2} \sin \theta_{x o}, y_{a}=v \cdot s_{y}^{2} \sin \theta_{y o}$ and $\mathcal{F} I_{2}(u, v)=\mathcal{F}_{\theta_{x o}, \theta_{y o}}\left[I_{2}\right]\left(u \cdot s_{x}^{2} \sin \theta_{x o}, v \cdot s_{y}^{2} \sin \theta_{y o}\right)$. The symbol $* *_{\text {conv }}$ denotes $2 \mathrm{D}$ spatial convolution. Eq. (38) shows that the reconstructed image of the disk is convolved by a Gaussian function. The Fourier transformation of the Jinc ${ }_{1}$-function is discussed in appendix (9) and it exhibits, in the case where the aperture of the filter is circular, the necessity to apply scale factors on the coordinates to retrieve the diameter $D_{t h}$. These scale factors, denoted by $\Delta_{j}$, are equal to:

$$
\Delta_{j}=\frac{|M|^{2}}{\pi^{2} L^{2}} \cdot \frac{\lambda_{m} \zeta_{e}}{s_{j}^{2} \sin \theta_{j o}},
$$

with $j=x, y$. In the previous theoretical example the scale factors are equal to $\Delta_{x}=\Delta_{y}=2.815$. In the case where the aperture of the filter is elliptical, the reconstruction give us, in first approximation, an autocorrelation of the disk function of radius 1 .

\subsection{Experimental results}

In order to validate the reconstruction process with the fractional Fourier transformation in practice, we have realized the experimental set-up illustrated in Figure 1). The intensity distribution of the interference between the incident beam and the part diffracted by the particle has been recorded and it is illustrated in Figure 2(b). Recall that the parameters are $\zeta_{s c}=296 \mathrm{~mm}, A=B=50 \mu \mathrm{m}$, and that the LED has mean wavelength $\lambda_{m}=625 \mathrm{~nm}$. The diameter of the particle is equal to $80 \mu \mathrm{m}$. The pixel size of the CCD sensor is equal to $4.4 \mu \mathrm{m}$ by $4.4 \mu \mathrm{m}$. In Figure $6(\mathrm{a})$, we have computed the reconstructed image of the opaque disk for optimal fractional orders $a_{x o}=a_{y o}=0.834$.

These experimental optimal fractional orders allow us to estimate the distance, denoted by $\zeta_{\text {est }}$, between the opaque disk and CCD sensor at $\zeta_{\text {est }}=80.33 \mathrm{~mm}$. In Figure 6(b), the intensity profile is given to demonstrate that the diameter of the particle can be estimated with an accuracy of 0.5 units.

The previous experimental results presented here are in good accordance with the expected results and validate our theoretical model of digital in-line holography with a spatially partially coherent beam.

\section{CONCLUSION}

To the best of our knowledge, we have proposed in this paper the first analytical solution to the problem of scalar diffraction of a partially coherent beam by an opaque disk. We have demonstrated that the expression for the intensity distribution in the spatially partially coherent beam is close to the 


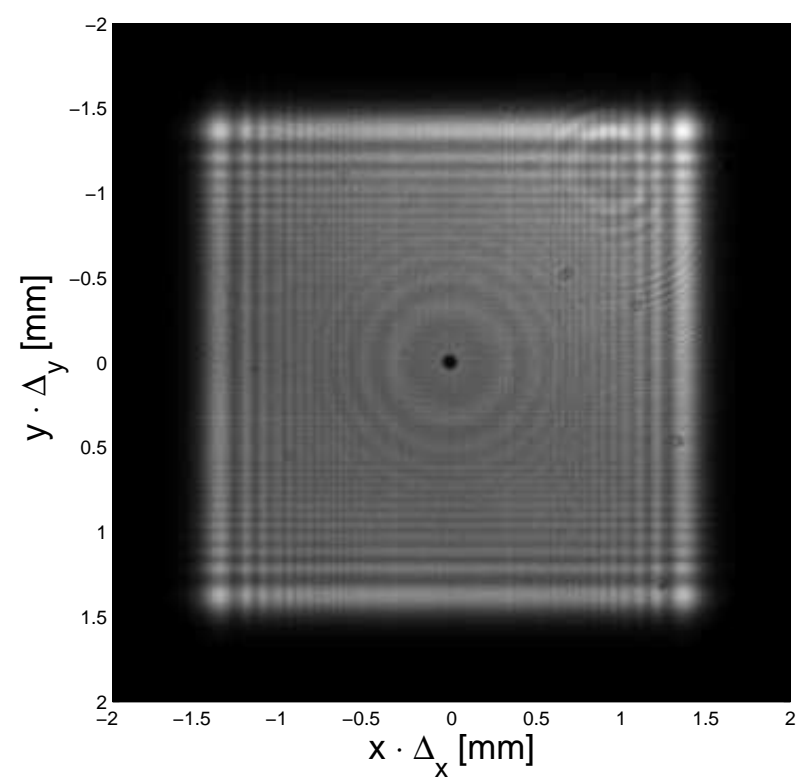

FIG. 6a Reconstruction of the image of the particle by the FRFT for fractional orders $a_{x o}=a_{y o}=0.834, \zeta_{\text {est }}=80.33 \mathrm{~mm}$.

expression of the intensity distribution in the case of a coherent beam. These expressions have been used in digital in-line holography. We have demonstrated that in the presence of a spatially partially coherent beam, the reconstruction process remains possible as in the case of a coherent beam. The optimal fractional orders that allow the reconstruction of the image of the opaque disk have been established theoretically in the case where the aperture of the filter is circular. We could demonstrate that there are fundamental differences in the reconstruction process when using a spatially partially coherent beam and coherent laser light. When using a spatially partially coherent beam, the orders of reconstruction and the diameter of the reconstructed object depend significantly on the source geometrie. The dependence of these parameters with the set-up could be established theoretically. Finally, digital in-line experiments have been carried out. A good agreement between the simulated intensity distributions and experimental results has been demonstrated and reconstructions could be performed by using the fractional Fourier transformation. They confirm the theoretical developments and predictions.

\section{APPENDIX}

\section{Definition of the functions $I(x, y)$}

\subsection{Expression for $I_{1}$}

The result of Eq. (7) is obtained by introducing Eq. (3) in Eq. (7). So

$$
\begin{aligned}
I_{1}(x, y)= & \frac{K}{\left(\lambda_{m} \zeta_{e}\right)^{2}} \int_{\mathbb{R}^{2}} \exp \left(-\frac{\pi^{2} A^{2}}{\left(\lambda_{m} \zeta_{s}\right)^{2}} \Delta \xi^{2}\right) \\
& \times \exp \left(-\frac{\pi^{2} B^{2}}{\left(\lambda_{m} \zeta_{s}\right)^{2}} \Delta v^{2}\right) \exp \left[i \frac{2 \pi}{\lambda_{m} \zeta_{e}}(x \Delta \xi+y \Delta v)\right] \\
& \times \int_{\mathbb{R}^{2}} \exp \left[-i 2 \pi\left(\xi\left(\frac{\Delta \xi}{\lambda_{m} \zeta_{e}}+\frac{\Delta \xi}{\lambda_{m} \zeta_{s}}\right)\right.\right. \\
& \left.\left.+v\left(\frac{\Delta v}{\lambda_{m} \zeta_{e}}+\frac{\Delta v}{\lambda_{m} \zeta_{s}}\right)\right)\right] d \xi d v d \Delta \xi d \Delta v, \\
= & \frac{K}{\left(L \lambda_{m} \zeta_{e}\right)^{2}} \int_{\mathbb{R}^{2}} \exp \left(-\frac{\pi^{2} A^{2}}{\left(\lambda_{m} \zeta_{s}\right)^{2}} \Delta \xi^{2}\right) \\
& \times \exp \left(-\frac{\pi^{2} B^{2}}{\left(\lambda_{m} \zeta_{s}\right)^{2}} \Delta v^{2}\right) \exp \left[i \frac{2 \pi}{\lambda_{m} \zeta_{e}}(x \Delta \xi+y \Delta v)\right] \\
& \times \delta(\Delta \xi, \Delta v) d \Delta \xi d \Delta v,
\end{aligned}
$$

with $\delta(x, y)$ the Dirac impulse and the constant $L=\left(\frac{1}{\lambda_{m} \zeta_{e}}+\frac{1}{\lambda_{m} \zeta_{s}}\right)$. From this one gets

$$
I_{1}(x, y)=\frac{A B}{\left(\zeta_{s}+\zeta_{e}\right)^{2}} \cdot I_{0} .
$$

\subsection{Expression for $I_{2}$}

Again, the result of Eq. (9) is obtained by introducing Eq. (3) in Eq. (9). So

$$
\begin{aligned}
I_{2}(x, y)= & \frac{K}{\left(\lambda_{m} \zeta_{e}\right)^{2}} \int_{\mathbb{R}^{4}} T\left(\xi-\frac{\Delta \xi}{2}, v-\frac{\Delta v}{2}\right) \\
& \exp \left(-\frac{\pi^{2} A^{2}}{\left(\lambda_{m} \zeta_{s}\right)^{2}} \Delta \xi^{2}\right) \exp \left(-\frac{\pi^{2} B^{2}}{\left(\lambda_{m} \zeta_{s}\right)^{2}} \Delta v^{2}\right) \\
& \exp \left[-i 2 \pi \frac{\xi \Delta \xi+v \Delta v}{\lambda_{m} \zeta_{s}}\right] \exp \left[-i \frac{2 \pi}{\lambda_{m} \zeta_{e}}(\xi \Delta \xi+v \Delta v)\right] \\
& \exp \left[i \frac{2 \pi}{\lambda_{m} \zeta_{e}}(x \Delta \xi+y \Delta v)\right] d \xi d v d \Delta \xi d \Delta v .
\end{aligned}
$$

To calculate this integral, three steps are necessary. The first step is to change variables $\xi-\Delta \xi / 2 \longrightarrow \xi^{\prime}, v-\Delta v / 2 \longrightarrow v^{\prime}$, and one gets in a straightforward manner:

$$
\begin{aligned}
I_{2}= & \frac{\pi K}{\left(\lambda_{m} \zeta_{e}\right)^{2} \sqrt{M N}} \cdot \exp \left[-\left(\alpha_{x} x^{2}+\alpha_{y} y^{2}\right)\right] \\
& \cdot \int_{\mathbb{R}^{2}} T\left(\xi^{\prime}, v^{\prime}\right) \cdot \exp \left[-\frac{\pi^{2} L^{2} \bar{M}}{|M|^{2}} \xi^{\prime 2}-\frac{\pi^{2} L^{2} \bar{N}}{|N|^{2}} v^{\prime 2}\right] \\
& \exp \left[-i \frac{2 \pi}{\lambda_{m} \zeta_{e}}\left(\frac{i \pi \bar{M} L}{|M|^{2}} x \xi^{\prime}+\frac{i \pi \bar{N} L}{|N|^{2}} y v^{\prime}\right)\right] d \xi^{\prime} d v^{\prime},
\end{aligned}
$$

with the coefficients

$$
\begin{aligned}
& M=\frac{\pi^{2} A^{2}}{\left(\lambda_{m} \zeta_{s}\right)^{2}}+i \pi L, \quad N=\frac{\pi^{2} B^{2}}{\left(\lambda_{m} \zeta_{s}\right)^{2}}+i \pi L, \\
& \alpha_{x}=\frac{\pi^{2}}{\left(\bar{\lambda} \zeta_{e}\right)^{2}} \frac{\bar{M}}{|M|^{2}}, \quad \alpha_{y}=\frac{\pi^{2}}{\left(\bar{\lambda} \zeta_{e}\right)^{2}} \frac{\bar{N}}{|N|^{2}} .
\end{aligned}
$$


The second step is to use polar coordinates $\xi^{\prime}=(1 / 2)$. $D \sigma \cos \theta$ and $\nu^{\prime}=(1 / 2) \cdot D \sigma \sin \theta$ in the integral in Eq. (43) to get

$$
\begin{aligned}
I_{2}= & \frac{\pi D_{t h}^{2}}{4} \frac{K}{\left(\lambda_{m} \zeta_{e}\right)^{2} \sqrt{M N}} \cdot \exp \left[-\left(\alpha_{x} x^{2}+\alpha_{y} y^{2}\right)\right] \\
& \times \int_{0}^{2 \pi} \int_{0}^{1} \exp \left[i \gamma \sigma^{2}\right] \exp \left[i \delta \sigma^{2} \cos (2 \theta)\right] \\
& \times \exp [i a \sigma \cos \theta+i b \sigma \sin \theta] \sigma d \sigma d \theta,
\end{aligned}
$$

with

$$
\begin{aligned}
& a=-i L \frac{\pi^{2} D_{t h}}{\lambda_{m} \zeta_{e}} \cdot \frac{\bar{M}}{|M|^{2}} \cdot x, \quad \text { and } \\
& b=-i L \frac{\pi^{2} D_{t h}}{\lambda_{m} \zeta_{e}} \cdot \frac{\bar{N}}{|N|^{2}} \cdot y .
\end{aligned}
$$

The parameters $\gamma, \delta$ are

$$
\begin{aligned}
& \gamma=i \pi^{2} \frac{D_{\text {th }}^{2}}{8}\left(\frac{\bar{M}}{|M|^{2}}+\frac{\bar{N}}{|N|^{2}}\right) L^{2}, \\
& \delta=i \pi^{2} \frac{D_{\text {th }}^{2}}{8}\left(\frac{\bar{M}}{|M|^{2}}-\frac{\bar{N}}{|N|^{2}}\right) L^{2} .
\end{aligned}
$$

By writing (see appendix 9 for comments)

$$
a \cos \theta+b \sin \theta=r \cos (\varphi-\theta),
$$

for which we have the condition

$$
a=r \cos \varphi, \quad b=r \sin \varphi,
$$

with complex $r$ and $\theta$, the integral in Eq. (45) has been evaluated in [12]. This gives then the result

$$
\begin{aligned}
I_{2}(r, \varphi)= & \frac{\pi^{2} K D_{t h}^{2}}{\left(\lambda \zeta_{e}\right)^{2} \sqrt{M N}} \cdot \exp \left[-\left(\alpha_{x} x^{2}+\alpha_{y} y^{2}\right)\right] \\
& \cdot \sum_{k=0}^{\infty}(-i)^{k} \varepsilon_{k} T_{k}(r, \gamma) \cos (2 k \varphi),
\end{aligned}
$$

with $\varepsilon_{k}=1 / 2$ if $k=0$ and 1 otherwise. The function $T_{k}(r, \gamma)$ is defined as

$$
T_{k}(r, \gamma)=\sum_{p=0}^{\infty} \beta_{2 k+2 p}^{2 k}(\delta) V_{2 k+2 p}^{2 k}(r, \gamma),
$$

where the expansion coefficients $\beta_{2 k+2 p}^{2 k}$ are expressed explicitly in terms of the hypergeometric functions ${ }_{2} F_{3}$ [12] as

$$
\begin{aligned}
& \beta_{2 k+2 p}^{2 k}\left(\gamma_{2}\right)=d_{0}^{0}(-1)^{r}(2 k+4 r+1)\left(\frac{1}{2} \gamma_{2}\right)^{k+2 r} \\
& { }_{2} F_{3}\left(\begin{array}{ccc}
r+\frac{1}{2} & k+r+\frac{1}{2} & \\
\frac{1}{2} & k+2 r+\frac{3}{2} & k+2 r+1
\end{array} ;-\frac{1}{4} \gamma_{2}^{2}\right)
\end{aligned}
$$

in the case where $2 r-p=0$ and

$$
\begin{aligned}
& \beta_{2 k+2 p}^{2 k}\left(\gamma_{2}\right)=d_{0}^{1}(-1)^{r}(2 k+4 r-1)\left(\frac{1}{2} \gamma_{2}\right)^{k+2 r} \\
& { }_{2} F_{3}\left(\begin{array}{ccc}
r+\frac{1}{2} & k+r+\frac{1}{2} & \\
\frac{3}{2}^{2} & k+2 r+1 & k+2 r+\frac{1}{2}
\end{array} ;-\frac{1}{4} \gamma_{2}^{2}\right)
\end{aligned}
$$

in the case where $2 r-p=1$. In Eqs. (52) and (54), the coefficients $d_{0}^{0}$ and $d_{0}^{1}$ are defined as follows:

$d_{0}^{0}=\frac{(2 r) !(2 k+2 r) !}{r !(k+r) !(2 k+4 r+1) !}, \quad d_{0}^{1}=\frac{(2 r) !(2 k+2 r) !}{r !(k+r) !(2 k+4 r) !}$.

\subsection{Expression for $I_{4}$}

The characteristic function of the unit disk is real and even and so the conjugate $\bar{T}$ of $T$ is equal to $T$. Therefore we must compute

$$
\begin{aligned}
I_{4}(x, y)= & \frac{1}{\left(\lambda_{m} \zeta_{e}\right)^{2}} \int_{\mathbb{R}^{4}} T\left(\xi-\frac{\Delta \xi}{2}, v-\frac{\Delta v}{2}\right) \\
& \times T\left(\xi+\frac{\Delta \xi}{2}, v+\frac{\Delta v}{2}\right) \widehat{J}_{o}(\xi, \Delta \xi, v, \Delta v) \\
& \times \exp \left[-i \frac{2 \pi}{\lambda_{m} \zeta_{e}}(\xi \Delta \xi+v \Delta v)\right] \\
& \times \exp \left[i \frac{2 \pi}{\lambda_{m} \zeta_{e}}(x \Delta \xi+y \Delta v)\right] d \xi d v d \Delta \xi d \Delta v
\end{aligned}
$$

Firstly, by changing variables $\xi-\Delta \xi / 2 \longrightarrow \xi^{\prime}, v-\Delta v / 2 \longrightarrow$ $v^{\prime}$ and by repeating the developments for $I_{2}$, the expression for $\mathrm{I}_{4}$ becomes

$$
\begin{aligned}
I_{4}= & \frac{K}{\left(\lambda_{m} \zeta_{e}\right)^{2}} \int_{\mathbb{R}^{2}} \exp \left[-M \Delta \xi^{2}-N \Delta v^{2}+i 2 \pi(X \Delta \xi+Y \Delta v)\right] \\
& \times\left(\int_{\mathbb{R}^{2}} T\left(\xi^{\prime}, v^{\prime}\right) T\left(\xi^{\prime}+\Delta \xi, v^{\prime}+\Delta v\right)\right. \\
& \left.\exp \left[-i 2 \pi L\left(\Delta \xi \cdot \xi^{\prime}+\Delta v \cdot v^{\prime}\right)\right] d \xi^{\prime} d v^{\prime}\right) d \Delta \xi d \Delta v
\end{aligned}
$$

with $X=x /\left(\lambda \zeta_{e}\right)$ and $Y=y /\left(\lambda \zeta_{e}\right)$. Changing variables $\xi^{\prime \prime}=\xi^{\prime} /\left(D_{t h} / 2\right), \Delta \xi^{\prime}=\Delta \xi /\left(D_{t h} / 2\right), v^{\prime \prime}=v^{\prime} /\left(D_{t h} / 2\right), \Delta v^{\prime}=$ $\Delta v /\left(D_{t h} / 2\right)$ and by noting that $\widetilde{T}\left(\xi^{\prime \prime}, v^{\prime \prime}\right)=T\left(\frac{D_{t h}}{2} \xi^{\prime \prime}, \frac{D_{t h}}{2} v^{\prime \prime}\right)$, we get

$$
\begin{aligned}
& I_{4}=\frac{D_{t h}^{4}}{16} \frac{K}{\left(\lambda_{m} \zeta_{e}\right)^{2}} \\
& \int_{\mathbb{R}^{2}} \exp \left[-\frac{M D_{t h}^{2}}{4} \Delta \xi^{\prime 2}-\frac{N D_{t h}^{2}}{4} \Delta v^{\prime 2}+i 2 \pi \frac{D_{t h}}{2}\left(X \Delta \xi^{\prime}+Y \Delta v^{\prime}\right)\right] \\
& \times\left(\int_{\mathbb{R}^{2}} \widetilde{T}\left(\xi^{\prime \prime}, v^{\prime \prime}\right) \widetilde{T}\left(\xi^{\prime \prime}+\Delta \xi^{\prime}, v^{\prime \prime}+\Delta v^{\prime}\right)\right. \\
& \left.\times \exp \left[-i 2 \pi \frac{L D_{t h}^{2}}{4}\left(\Delta \xi^{\prime} \cdot \xi^{\prime \prime}+\Delta v^{\prime} \cdot v^{\prime \prime}\right)\right] d \xi^{\prime \prime} d v^{\prime \prime}\right) d \Delta \xi^{\prime} d \Delta v^{\prime} .
\end{aligned}
$$

Now, by setting $\widetilde{T}(\sigma)=\widetilde{T}\left(\Delta \xi^{\prime}, \Delta v^{\prime}\right)$ where $\sigma=\left(\Delta \xi^{\prime}, \Delta v^{\prime}\right)$, we have

$$
\begin{aligned}
& \left(\widetilde{T}(\sigma) \exp \left[-i \pi \frac{L D_{t h}^{2}}{4} \sigma \cdot \sigma\right]_{\text {corr }}^{\star \star} \widetilde{T}(\sigma)\right. \\
& \left.\exp \left[-i \pi \frac{L D_{t h}^{2}}{4} \sigma \cdot \sigma\right]\right) \times\left(\Delta \xi^{\prime}, \Delta v^{\prime}\right) \\
= & \exp \left[-i \pi \frac{L D_{t h}^{2}}{4}\left(\Delta \xi^{\prime 2}+\Delta v^{\prime 2}\right)\right] \\
& \int_{\mathbb{R}^{2}} \widetilde{T}\left(\xi^{\prime \prime}, v^{\prime \prime}\right) \widetilde{T}\left(\xi^{\prime \prime}+\Delta \xi^{\prime}, v^{\prime \prime}+\Delta v^{\prime}\right) \\
& \times \exp \left[-i 2 \pi \frac{L D_{t h}^{2}}{4}\left(\xi^{\prime \prime} \Delta \xi^{\prime}+v^{\prime \prime} \Delta v^{\prime}\right)\right] d \xi^{\prime \prime} d v^{\prime \prime},
\end{aligned}
$$


where $\star \star$ corr denotes 2-D spatial correlation. Hence Eq. (59) for $I_{4}$ becomes

$$
\begin{aligned}
I_{4}= & \frac{D_{t h}^{4}}{16} \frac{K}{\left(\lambda_{m} \zeta_{e}\right)^{2}} \int_{\mathbb{R}^{2}} \times \exp \left[-\frac{M D_{t h}^{2}}{4} \Delta \xi^{\prime 2}-\frac{N D^{2}}{4} \Delta v^{\prime 2}\right] \\
& \times \exp \left[i 2 \pi \frac{D_{t h}}{2}\left(X \Delta \xi^{\prime}+Y \Delta v^{\prime}\right)\right] \\
& \times \exp \left[i \pi \frac{L D_{t h}^{2}}{4}\left(\Delta \xi^{\prime 2}+\Delta v^{\prime 2}\right)\right] \\
& \times\left(\widetilde{T}(\sigma) \exp \left[-i \pi L \frac{D_{t h}^{2}}{4} \sigma \cdot \sigma\right] \underset{\text { corr }}{\star \star}(\sigma)\right. \\
& \left.\times \exp \left[-i \pi L \frac{D_{t h}^{2}}{4} \sigma \cdot \sigma\right]\right)\left(\Delta \xi^{\prime}, \Delta v^{\prime}\right) \cdot d \Delta \xi^{\prime} d \Delta v^{\prime} .
\end{aligned}
$$

From the definition of the coefficients $M$ and $N$, the integral $I_{4}$ can be written as

$$
\begin{aligned}
I_{4}= & \frac{D_{t h}^{4}}{16} \frac{K}{\left(\lambda_{m} \zeta_{e}\right)^{2}} \\
& \times \int_{\mathbb{R}^{2}} \exp \left[-\mathcal{A} \Delta \xi^{\prime 2}-\mathcal{B} \Delta v^{\prime 2}+i 2 \pi\left(\mathcal{X} \Delta \xi^{\prime}+\mathcal{Y} \Delta v^{\prime}\right)\right] \\
& \times(\widetilde{T}(\sigma) \exp [-i \pi \kappa \sigma \cdot \sigma] \underset{\text { corr }}{\star \star} \widetilde{T}(\sigma) \exp [-i \pi \kappa \sigma \cdot \sigma]) \\
& \times\left(\Delta \xi^{\prime}, \Delta v^{\prime}\right) \cdot d \Delta \xi^{\prime} d \Delta v^{\prime},
\end{aligned}
$$

with

$$
\begin{gathered}
\mathcal{A}=\frac{\pi^{2} D_{t h}^{2}}{4} \frac{A^{2}}{\left(\lambda \zeta_{s}\right)^{2}}, \quad \mathcal{B}=\frac{\pi^{2} D_{t h}^{2}}{4} \frac{B^{2}}{\left(\lambda \zeta_{s}\right)^{2}}, \\
\kappa=\frac{L D_{t h}^{2}}{4}, \quad \mathcal{X}=\frac{D_{t h}}{2 \lambda \zeta_{e}} \cdot x, \quad \mathcal{Y}=\frac{D_{t h}}{2 \lambda \zeta_{e}} \cdot y .
\end{gathered}
$$

The variables in the integral in Eq. (62) are dimensionless and will be used in the sequel. To compute the autocorrelation in Eq. (62), the well-known Zernike expansion of the function $\widetilde{T}(\sigma) \exp [-i \pi \kappa \sigma \cdot \sigma]$ is used. We have only to consider $R_{2 l}^{0}(\sigma)$ by radial symmetry and by using Bauer's identity, see, for instance [31], Eq. (8), we have

$$
\widetilde{T}(\sigma) \exp [-i \pi \kappa \sigma \cdot \sigma]=\sum_{l=0}^{\infty} \mathcal{B}_{2 l}^{0}(\kappa) \cdot R_{2 l}^{0}(\sigma),
$$

where

$$
\mathcal{B}_{2 l}^{0}(\kappa)=(2 l+1) \exp \left[-i \frac{\pi \kappa}{2}\right](-i)^{l} \cdot j_{l}\left(\frac{\pi \kappa}{2}\right),
$$

with $j_{l}$ the spherical Bessel functions of the first kind. Accordingly, the autocorrelation of the function $\widetilde{T}(\sigma) \exp [-i \pi \kappa \sigma \cdot \sigma]$ can be expressed as

$$
\begin{aligned}
& \widetilde{T}(\sigma) \exp [-i \pi \kappa \sigma \cdot \sigma]_{\text {corr }}^{\star \star} \underset{T}{T}(\sigma) \exp [-i \pi \kappa \sigma \cdot \sigma] \\
= & \sum_{l, l^{\prime}}^{\infty} \mathcal{B}_{2 l}^{0}(\kappa) \overline{\mathcal{B}_{2 l^{\prime}}^{0}(\kappa)}\left[R_{2 l}^{0}(\sigma)_{\text {corr }}^{\star \star} R_{2 l^{\prime}}^{0}(\sigma)\right] .
\end{aligned}
$$

From [30], the correlation of two Zernike polynomials has the Zernike expansion

$$
\begin{aligned}
R_{2 l}^{0}(\sigma) \underset{\text { corr }}{\star \star} R_{2 l^{\prime}}^{0}(\sigma)= & \frac{\pi / 4}{(2 l+1)\left(2 l^{\prime}+1\right)} \\
& \times \sum_{q=0}^{\infty}(2 q+1) \cdot \Gamma_{2 l, 2 l^{\prime}, 2 q}^{000} \cdot R_{2 q}^{0}\left(\frac{\sigma}{2}\right)
\end{aligned}
$$

Note that we want $R_{2 q}^{0}\left(\frac{\sigma}{2}\right)$ rather than $R_{2 q}^{0}(\sigma)$ since the correlation of two Zernike terms is non-vanishing for $\sigma \leq 2$. The expansion coefficients $\Gamma_{2 l, 2 l^{\prime}, 2 p}^{000}$ are expressed in [30] in terms of at most four Jacobi polynomials according to

$$
\begin{aligned}
\Gamma_{2 l, 2 l^{\prime}, 2 q}^{000}= & 2(-1)^{\frac{2 l+2 l^{\prime}+2 q}{2}} \\
& \cdot\left[Q_{2 l, 2 l^{\prime}}^{2 q+1}+Q_{2 l+2,2 l^{\prime}}^{2 q+1}+Q_{2 l, 2 l^{\prime}+2}^{2 q+1}+Q_{2 l+2,2 l^{\prime}+2}^{2 q+1}\right],
\end{aligned}
$$

with

$$
\begin{aligned}
& Q_{i, j}^{n^{\prime \prime}+1} \\
& =\left\{\begin{array}{l}
\frac{\left(\frac{1}{2}\left(n^{\prime \prime}+i+j\right)\right) !\left(\frac{1}{2}\left(n^{\prime \prime}-i-j\right)\right) !}{\left(\frac{1}{2}\left(n^{\prime \prime}+i-j\right)\right) !\left(\frac{1}{2}\left(n^{\prime \prime}+j-i\right)\right) !} \cdot\left(\frac{1}{2}\right)^{i+j+1} \cdot P_{\frac{n^{\prime \prime}-i-j}{2}}^{(i, j)}(0) \cdot P_{\frac{n^{\prime \prime}-i-j}{2}}^{(j, i)}(0), \\
0
\end{array}\right. \\
& \text { if } n^{\prime \prime} \geq i+j \text {, } \\
& \text { otherwise. }
\end{aligned}
$$

and $P$ the general Jacobi polynomial. We can conclude that the autocorrelation takes the form:

$$
\begin{aligned}
& \widetilde{T}(\sigma) \exp [-i \pi \kappa \sigma \cdot \sigma]_{\text {corr }}^{\star \star} \widetilde{T}(\sigma) \exp [-i \pi \kappa \sigma \cdot \sigma] \\
= & \frac{\pi}{4} \sum_{q=0}^{\infty} C_{q}(\kappa) \cdot R_{2 q}^{0}\left(\frac{\sigma}{2}\right),
\end{aligned}
$$

with

$$
C_{q}(\kappa)=\sum_{l, l^{\prime}}^{\infty} \frac{(2 q+1) \Gamma_{2 l, 2 l^{\prime}, 2 q}^{000}}{(2 l+1)\left(2 l^{\prime}+1\right)} \mathcal{B}_{2 l}^{0}(\kappa) \overline{\mathcal{B}_{2 l^{\prime}}^{0}(\kappa)} .
$$

Consequently by combining Eqs. (70) and (62)

$$
\begin{aligned}
I_{4}= & \frac{\pi D_{t h}^{4}}{64} \frac{K}{\left(\lambda_{m} \zeta_{e}\right)^{2}} \sum_{q=0}^{\infty} C_{q}(\mathcal{K}) \\
& \times \int_{\mathbb{R}^{2}} \exp \left[-\mathcal{A} \Delta \xi^{\prime 2}-\mathcal{B} \Delta v^{\prime 2}+i 2 \pi\left(\mathcal{X} \Delta \xi^{\prime}+\mathcal{Y} \Delta v^{\prime}\right)\right] \\
& \cdot R_{2 q}^{0}\left(\frac{\sigma}{2}\right) \cdot d \Delta \xi^{\prime} d \Delta v^{\prime} .
\end{aligned}
$$

To get a semi-analytical computation method for the remaining integral in Eq. (72), the approach is as follows. Firstly, write

$$
\begin{aligned}
& \exp \left[-\mathcal{A} \Delta \xi^{\prime 2}-\mathcal{B} \Delta v^{\prime 2}\right] \\
= & \exp \left[-\frac{1}{2}(\mathcal{A}+\mathcal{B}) \sigma^{2}\right] \cdot \exp \left[-\frac{1}{2}(\mathcal{A}-\mathcal{B}) \sigma^{2} \cos (2 \theta)\right],
\end{aligned}
$$

where $\Delta \xi^{\prime}+i \Delta v^{\prime}=\sigma \exp (i \theta)$. Then expand, using $\beta$ coefficients as earlier

$$
\begin{aligned}
& \exp \left[-\frac{1}{2}(\mathcal{A}-\mathcal{B}) \sigma^{2} \cos (2 \theta)\right] \\
= & \sum_{n=-\infty}^{+\infty} \sum_{p=0}^{\infty} i^{n} \cdot \beta_{|2 n|+2 p}^{|2 n|}(\psi) \cdot R_{|2 n|+2 p}^{|2 n|}\left(\frac{\sigma}{2}\right) \exp (i 2 n \theta),
\end{aligned}
$$

with $\psi=2 i(\mathcal{A}-\mathcal{B})$. The coefficients $\beta$ are expressed explicitly in terms of the hypergeometric functions ${ }_{2} F_{3}$ as in [12], see Eqs. (A-11)-(A-13). Note that, we want $R\left(\frac{1}{2} \sigma\right)$ rather than $R(\sigma)$, see Eq. (70). When Eq. (74) is introduced in Eq. (72), we see that the product of two Zernike polynomials

$$
R_{2 q}^{0}\left(\frac{\sigma}{2}\right) \cdot R_{|2 n|+2 p}^{|2 n|}\left(\frac{\sigma}{2}\right)
$$


arises. In [31] these products are linearized, but in [32] there occurs an easier expression in terms of Wigner coefficients (or Clebsch-Gordan coefficients), see Eq. (27.9.1) on p.1006 of [27]. Thus we have

$$
\begin{aligned}
& R_{2 q}^{0}\left(\frac{\sigma}{2}\right) \cdot R_{|2 n|+2 p}^{|2 n|}\left(\frac{\sigma}{2}\right) \\
= & \sum_{s=\max (0, p-q, q-p-|2 n|)}^{p+q} A_{|2 n|+2 p, 2 q,|2 n|+2 s}^{|2 n|, 0,2 n \mid} \cdot R_{|2 n|+2 s}^{|2 n|}\left(\frac{\sigma}{2}\right),
\end{aligned}
$$

with

$$
A_{|2 n|+2 p, 2 q,|2 n|+2 s}^{|2 n|, 0,|2 n|}=\left(C_{\frac{|2 n|}{2}, 0, \frac{|2 n|+2 p}{2}}^{\frac{\mid 2 \eta}{2}, \frac{|2 n|+2 s}{2}}\right)^{2} .
$$

With steps one and two, the integral in question is now given as

$$
\begin{aligned}
& \int_{0}^{2} \int_{0}^{2 \pi} R_{|2 n|+2 s}^{|2 n|}\left(\frac{\sigma}{2}\right) \exp [i 2 n \theta] \exp \left[-\frac{1}{2}(\mathcal{A}+\mathcal{B}) \sigma^{2}\right] \\
& \times \exp [i 2 \pi(\mathcal{X} \cos (\theta)+\mathcal{Y} \sin (\theta)) \sigma] \sigma d \sigma d \theta .
\end{aligned}
$$

This latter integral can be expressed in term of V-functions from the extended Nijboer-Zernike theory $[12,29]$. Indeed, as we have integers $|2 n|$ and $|2 n|+2 s$ and $|2 n|+2 s-|2 n|=2 s$ even and non-negative, we have

$$
\begin{aligned}
& \int_{0}^{2} \int_{0}^{2 \pi} R_{|2 n|+2 s}^{|2 n|}\left(\frac{\sigma}{2}\right) \exp [i 2 n \theta] \exp \left[-\frac{1}{2}(\mathcal{A}+\mathcal{B}) \sigma^{2}\right] \\
& \quad \times \exp [i 2 \pi(\mathcal{X} \cos (\theta)+\mathcal{Y} \sin (\theta)) \sigma] \sigma d \sigma d \theta \\
& =8 \pi(-1)^{n} \exp [i 2 n \varphi] \cdot V_{|2 n|+2 s}^{|2 n|}(4 \pi r, \chi),
\end{aligned}
$$

where $\mathcal{X}+i \mathcal{Y}=r \exp (i \varphi), \chi=2 i(\mathcal{A}+\mathcal{B})$ and the powerBessel series is given by Eq. (18). This finally results into the the semi-analytical formula for the integral $I_{4}$ that we were looking for:

$$
\begin{aligned}
I_{4}(r, \varphi)= & \frac{\pi^{2} D_{t h}^{4}}{8} \frac{K}{\left(\lambda_{m} \zeta_{e}\right)^{2}} \sum_{q=0}^{\infty} C_{q}(\kappa) \sum_{n=-\infty}^{\infty} \sum_{p=0}^{\infty} i^{n} \beta_{|2 n|+2 p}^{|2 n|}(\psi) \\
& \times \sum_{s=\max (0, p-q, q-p-|2 n|)}^{p+q} A_{|2 n|+2 p, 2 q,|2 n|+2 s}^{|2 n|, 0,2 n \mid} V_{|2 n|+2 s}^{|2 n|} \\
& \times(4 \pi r, \chi) \exp [i 2 n \varphi]
\end{aligned}
$$

\section{Comments about conditions}

\section{Eq. (13)}

Given complex numbers $a, b$ such that $a+i b \neq 0 \neq a-i b$, it is required to find (complex) $r$ and $\varphi$ such that

$$
a=r \cos \varphi, \quad b=r \sin \varphi .
$$

Eq. (81) is equivalent with

$$
\begin{aligned}
& z:=a+i b=r \cos \varphi+i r \sin \varphi=r \exp (i \varphi), \\
& w:=a-i b=r \cos \varphi-i r \sin \varphi=r \exp (-i \varphi) .
\end{aligned}
$$

Thus, given two complex numbers $z \neq 0 \neq w$, we want to find $r$ and $s$ such that

$$
r s=z, \quad r / s=w .
$$

To that end, we let $r$ be one of the square roots of $z w$, so $r^{2}=z w$, and we let $s=z / r$. Then $r \neq 0 \neq s$, and it is verified that Eq. (83) holds. Finally, we must choose $\varphi$ (complex) such that $s=\exp (i \varphi)$.

\section{Reconstruction of the image of the particle}

Formally, the Fourier transformation of the Jinc ${ }_{1}$-function is expressed, using polar coordinates $x=\rho \cos \theta, y=\rho \sin \theta$ and $u=\rho_{a} \cos \theta_{a}, v=\rho_{a} \sin \theta_{a}$, as

$\mathcal{F}\left[\frac{J_{1}(r)}{r}\right]=\int_{0}^{+\infty} \int_{0}^{2 \pi} \frac{J_{1}(r)}{r} \cdot \exp \left[-i 2 \pi \rho \rho_{a} \cos \left(\theta-\theta_{a}\right)\right] \rho d \rho d \theta$,

where

$$
r^{2}=\frac{1}{2} \rho^{2} \cdot\left[a^{\prime 2}+b^{\prime 2}+\left(a^{\prime 2}-b^{\prime 2}\right) \cos (2 \theta)\right],
$$

with

$$
a^{\prime}=-i L \frac{\pi^{2} D_{t h}}{\lambda_{m} \zeta_{e}} \cdot \frac{\bar{M}}{|M|^{2}}, \quad b^{\prime}=-i L \frac{\pi^{2} D_{t h}}{\lambda_{m} \zeta_{e}} \cdot \frac{\bar{N}}{|N|^{2}} .
$$

Now, to propose an evaluation of Eq. (84), the Gegenbauer's addition theorem is used, see [27], Eq. (9.1.80) on p.363,

$$
\begin{aligned}
\frac{\mathcal{C}_{v}(\omega)}{\omega^{v}}= & 2^{v} \Gamma(v) \cdot \sum_{k=0}^{\infty}(v+k) \\
& \cdot \frac{\mathcal{C}_{v+k}(U)}{U^{v}} \cdot \frac{J_{v+k}(V)}{V^{v}} \cdot C_{k}^{(v)}(\cos \phi),
\end{aligned}
$$

where $\omega=\left(U^{2}+V^{2}-2 U V \cos \phi\right)^{1 / 2}$ and where $C_{m}^{(1)}$ represents the Gegenbauer's polynomial involving trigonometric function, for $\mathcal{C}=J, v=1$. By considering the variables:

$$
U=\frac{1}{2} \rho\left(b^{\prime}-a^{\prime}\right) \quad V=\frac{1}{2} \rho\left(b^{\prime}+a^{\prime}\right),
$$

we obtain

$$
r=\sqrt{U^{2}+V^{2}-2 U V \cos (2 \theta)} .
$$

With this definition of $r$ and Eq. (87), the Jinc ${ }_{1}$-function can be expressed as

$$
\frac{J_{1}(r)}{r}=2 \sum_{m=0}^{\infty}(m+1) \frac{J_{m+1}(U)}{U} \cdot \frac{J_{m+1}(V)}{V} \cdot C_{m}^{(1)}(\cos (2 \theta)) .
$$

Now $r=r(x, y)$ is complex and $J_{1}(r) / r$ decays only along the real axis while $J_{1}(r) / r$ grows exponentially in any other direction. Hence its Fourier transform is not defined. We get around this problem as follows. It is seen from Eqs. (88) and (90) that $J_{1}(r) / r$ depends analytically on $a^{\prime}$ and $b^{\prime}$ (Eq. (90) converges rapidly, also in case of complex $U$ and $V$ ). Therefore, the integral expression in Eq. (36) depends analytically on $a^{\prime}$ and $b^{\prime}$. If we consider that the imaginary parts of $a^{\prime}$ and $b^{\prime}$ are small, we could use in Eq. (36) $J_{1}(r) / r$, with $a^{\prime}, b^{\prime}$ replaced by their real parts $\Re\left(a^{\prime}\right)$ and $\Re\left(b^{\prime}\right)$. Then $U$ and $V$ in Eq. (88) are real, and we obtain from Eqs. (84) and (88) that, for evaluation of Eq. (36), we can use

$$
\begin{aligned}
& \mathcal{F}\left[\frac{J_{1}(r)}{r}\right] \approx 2 \sum_{m=0}^{\infty}(m+1) \int_{0}^{+\infty} \frac{J_{m+1}(U)}{U} \cdot \frac{J_{m+1}(V)}{V} \\
& \times \int_{0}^{2 \pi} C_{m}^{(1)}(\cos (2 \theta)) \cdot \exp \left[-i 2 \pi \rho \rho_{a} \cos \left(\theta-\theta_{a}\right)\right] d \theta \cdot \rho d \rho .
\end{aligned}
$$

By using [27], Eq. (22.312) on p.776,

$$
C_{m}^{(1)}(\cos (2 \theta))=\sum_{k=0}^{m} \cos [2(m-2 k) \theta],
$$


then

$$
\begin{aligned}
& \int_{0}^{2 \pi} C_{m}^{(1)}(\cos (2 \theta)) \exp \left[-i 2 \pi \rho \rho_{a} \cos \left(\theta-\theta_{a}\right)\right] d \theta \\
= & 2 \pi \sum_{k=0}^{m}(-1)^{m-2 k} \cdot J_{2 m-4 k}\left(2 \pi \rho \rho_{a}\right) \cdot \cos \left(2(m-2 k) \theta_{a}\right) .
\end{aligned}
$$

Consequently,

$$
\begin{aligned}
\mathcal{F}\left[\frac{J_{1}(r)}{r}\right] & \approx 4 \pi \sum_{m=0}^{\infty}(m+1) \sum_{k=0}^{m} \cos \left(4 k \theta_{a}\right) \\
& \cdot \int_{0}^{+\infty} \frac{J_{m+1}(U)}{U} \cdot \frac{J_{m+1}(V)}{V} J_{2 m-4 k}\left(2 \pi \rho \rho_{a}\right) \rho d \rho
\end{aligned}
$$

Now, we can discuss about Eq. (94) for two particular cases. The case where the filter is circular and the case where it is elliptical.

\subsection{First case: circular filter, $A=B$}

In this case, $a^{\prime}=b^{\prime}$ and $\Re\left(a^{\prime}\right)=\Re\left(b^{\prime}\right)$ thus $U=0$ and $V=a^{\prime} \rho$. For $(m+1)$ fixed and $U=0, \frac{J_{m+1}(U)}{U}$ is equal to $1 / 2$ if $m=0$ and zero otherwise, thus

$$
\mathcal{F}\left[\frac{J_{1}(r)}{r}\right]=\frac{2 \pi}{a^{\prime}} \int_{0}^{+\infty} J_{1}\left(a^{\prime} \rho\right) J_{0}\left(2 \pi \rho \rho_{a}\right) d \rho .
$$

As we have considered $a^{\prime} \approx \Re\left(a^{\prime}\right)$, for the purpose of evaluating the integral in Eq. (36), we can replace Eq. (95) by

$$
\left.\mathcal{F}\left[\frac{J_{1}(r)}{r}\right] \approx \frac{2 \pi}{\Re\left(a^{\prime}\right)} \int_{0}^{+\infty} J_{1}\left(\Re\left(a^{\prime}\right) \rho\right)\right) J_{0}\left(2 \pi \rho \rho_{a}\right) d \rho .
$$

With the discontinuous Weber-Schafheitlin integral in [27] 11.4.42 on p.487, we have:

$$
\mathcal{F}\left[\frac{J_{1}(r)}{r}\right] \approx \frac{2 \pi}{\Re\left(a^{\prime}\right)} \times \begin{cases}1, & \text { if } 2 \pi \sqrt{u^{2}+v^{2}}<\left|\Re\left(a^{\prime}\right)\right|, \\ 0, & \text { otherwise. }\end{cases}
$$

The condition of (97) allows us to define the scale factor to apply on the axes to retrieve the diameter of the opaque disk. Indeed, with the definition of the frequencies $(u, v)$, we have the inequality

$$
\left(x_{a}^{2}+y_{a}^{2}\right)^{1 / 2}<\frac{\left|\Re\left(a^{\prime}\right)\right|}{2 \pi} \cdot s_{x}^{2} \sin \left(\theta_{x o}\right) .
$$

with

$$
\left|\Re\left(a^{\prime}\right)\right|=\frac{\pi^{3} L^{2}}{\lambda_{m} \zeta_{e}} \cdot \frac{D_{t h}}{|M|^{2}} .
$$

The estimated diameter, denoted $D_{\text {est }}$, in the reconstructed image of the opaque disk is then:

$$
D_{e s t}=\frac{\pi^{2} L^{2}}{\lambda_{m} \zeta_{e}} \cdot \frac{s_{x}^{2} \sin \left(\theta_{x o}\right)}{|M|^{2}} \cdot D_{t h} .
$$

In conclusion, the scale factor to apply on the axes is then

$$
\Delta_{x}=\frac{\lambda_{m} \zeta_{e}}{\pi^{2} L^{2}} \cdot \frac{|M|^{2}}{s_{x}^{2} \sin \left(\theta_{x o}\right)}, \quad \Delta_{y}=\frac{\lambda_{m} \zeta_{e}}{\pi^{2} L^{2}} \cdot \frac{|N|^{2}}{s_{y}^{2} \sin \left(\theta_{y o}\right)},
$$

where $N=M, \theta_{x o}=\theta_{y o}$ and $s_{x}=s_{y}$ to retrieve the real diameter of the opaque disk.

\subsection{Second case: elliptical filter, $A \neq B$}

In first approximation, $m=k=0$, and using the relations (88), we have

$$
\mathcal{F}\left[\frac{J_{1}(r)}{r}\right] \approx 4 \pi \int_{0}^{+\infty} \frac{J_{1}(U)}{U} \cdot \frac{J_{1}(V)}{V} J_{0}\left(2 \pi \rho \rho_{a}\right) \rho d \rho .
$$

As previously mentioned, the imaginary parts of $b^{\prime}-a^{\prime}$ and $b^{\prime}+a^{\prime}$ are small. Furthermore $\Re\left(b^{\prime}-a^{\prime}\right) \approx \Re\left(b^{\prime}+a^{\prime}\right)$ and thus $U \approx V=\frac{1}{2} \rho \Re\left(b^{\prime}+a^{\prime}\right)$. Then Eq. (102) becomes

$$
\begin{aligned}
\mathcal{F}\left[\frac{J_{1}(r)}{r}\right] \approx & \frac{16 \pi}{\Re\left(b^{\prime}+a^{\prime}\right)^{2}} \int_{0}^{+\infty} J_{1}\left(\frac{1}{2} \rho \Re\left(b^{\prime}+a^{\prime}\right)\right) \\
& \cdot J_{1}\left(\frac{1}{2} \rho \Re\left(b^{\prime}+a^{\prime}\right)\right) J_{0}\left(2 \pi \rho \rho_{a}\right) \frac{1}{\rho} d \rho .
\end{aligned}
$$

Finally, from [30], Eq. (60), the integral at the right-hand side of Eq. (103) can be evaluated, and we obtain

$$
\mathcal{F}\left[\frac{J_{1}(r)}{r}\right] \approx \frac{16 \pi}{\Re\left(b^{\prime}+a^{\prime}\right)^{2}}\left(T_{\text {corr }}^{\star \star} T\right)\left(4 \pi \frac{\rho_{a}}{\Re\left(b^{\prime}+a^{\prime}\right)}\right),
$$

where $\star \star \star_{\text {corr }}$ denotes the $2 \mathrm{D}$-correlation product and $T$ is the indicator function of the disk of radius 1 .

\section{ACKNOWLEDGEMENTS}

The authors thank F. David, J.M. Dorey, EDF R \& D, Mécanique des Fluides, Energies, Environnement, Chatou, France, for supporting this study.

\section{References}

[1] J.-M. Desse, P. Picart, and P. Tankam, "Digital three-color holographic interferometry for flow analysis" Opt. Express 16, 5471-5480 (2008).

[2] 0. Kafri, and I. Glatt, The Physics of Moiré Metrology (Wiley, New York, 1989).

[3] F. Slimani, G. Grehan, G. Gouesbet, and D. Allano, "Near-field Lorenz-Mie theory and its application to microholography" Appl. Opt. 23, 4140-4148 (1984).

[4] T. Kreis, Handbook of Holographic Interferometry: Optical and Digital Methods (Wiley-VCH Verlag CmbH At Co. KGaA, 2004).

[5] F. Nicolas, S. Coëtmellec, M. Brunel, and D. Lebrun, “Digital in-line holography with a sub-picosecond laser beam" Opt. Commun. 268, 27-33 (2006).

[6] L. Mandel, and E. Wolf, Optical Coherence and Quantum optics (Cambridge U. Press, Cambridge, 1995).

[7] H. T. Eyyuboğlu, Y. Baykal, and Y. Cai, “Complex degree of coherence for partially coherent general beams in atmospheric turbulence" J. Opt. Soc. Am. A 24, 2891-2901 (2007).

[8] D. Gabor, "A new microscopic principle" Nature 161, 777-778 (1948).

[9] J. W. Goodman, Introduction to Fourier Optics, $3^{\text {rd }}$ ed. (Roberts and Company Publishers, Greenwood village, USA, 2005).

[10] U. Schnars, and W. Jüptner, "Direct recording of holograms by a CCD target and numerical reconstruction" Appl. 0pt. 33, 179-181 (1994). 
[11] D. J. Stigliani, JR., R. Mittra, and R. G. Semonin, "Particle-Size Measurement Using Forward-Scatter Holography" J. Opt. Soc. Am. 60, 1059-1067 (1970)

[12] F. Nicolas, S. Coëtmellec, M. Brunel, D. Allano, D. Lebrun, and A. J. E. M. Janssen, "Application of the fractional Fourier transformation to digital holography recorded by an elliptical, astigmatic Gaussian beam" J. Opt. Soc. Am. A 22, 2569-2577 (2005).

[13] J. J. Wen, and M. Breazeale, "Gaussian beam functions as a base function set for acoustical field calculations" in Proceedings to IEEE Ultrasonics Symposium 1137-1140 (IEEE, Denver, 1987).

[14] J. J. Wen, and M. Breazeale, "A diffraction beam expressed as the superposition of Gaussian beams," J. Acoust. Soc. Am. 83, 1752-1756 (1988).

[15] S. Coëtmellec, N. Verrier, M. Brunel, D. Lebrun, "General formulation of digital in-line holography from correlation with a chirplet function", J. Eur. Opt. Soc.-Rapid 5, 10027 (2010).

[16] F. Dubois, M. L. N. Requena, C. Minetti, 0. Monnom, and E. Istasse, "Partial spatial coherence effects in digital holographic microscopy with a laser source," Appl. Opt. 43, 1131-1139 (2004).

[17] J. W. Goodman, Statistical Optics (Wiley Classics Library, New York, 2000).

[18] A. E. Siegman, Lasers (University Science Books, Mill Valley, 1986).

[19] X. Du, and D. Zhao, "Propagation of elliptical Gaussian beams in apertured and misaligned optical systems," J. Opt. Soc. Am. A 23, 1946-1950 (2006).

[20] N. Verrier, S. Coëtmellec, M. Brunel, D. Lebrun, and A. J. E. M Janssen, "Digital in-line holography with an elliptical, astigmatic Gaussian beam: wide-angle reconstruction," J. Opt. Soc. Am. A 25, 1459-1466 (2008).

[21] F. Dubois, C. Schockaert, N. Callens, and C. Yourassowsky, "Focus plane detection criteria in digital holography microscopy by amplitude analysis," Opt. Express 14, 5895-5908 (2006).

[22] B. Ge, Q. Lu, and Y. Zhang, "Particle digital in-line holography with spherical wave recording", Chin. Opt. Lett. 01, 517 (2003).

[23] R. B. Owen, and A. A. Zozulya, "In-line digital holographic sensor for monitoring and characterizing marine particulates", Opt. Eng. 39, 2187 (2000).
[24] A. C. McBride, and F. H. Kerr, "On Namias's fractional Fourier transforms", IMA J. Appl. Math. 39, 159-175 (1987).

[25] V. Namias, "The fractional order Fourier transform and its application to quantum mechanics", J. Inst. Maths Its Applics, 25, 241-265 (1980).

[26] A. W. Lohmann, "Image rotation, Wigner rotation, and the fractional Fourier transform", J. Opt. Soc. Am. A 10, 2181-2186 (1993).

[27] M. Abramowitz, and I. A. Stegun, Handbook of Mathematical Functions (Dover Publications, Inc., New York, 1970).

[28] J. J. M. Braat, P. Dirksen, and A. J. E. M. Janssen, "Assessment of an extended Nijboer-Zernike approach for the computation of optical point-spread functions", J. Opt. Soc. Am. A 19, 858-870 (2002).

[29] A. J. E. M. Janssen, "Extended Nijboer-Zernike approach for the computation of optical point-spread functions", J. Opt. Soc. Am. A 19, 849-857 (2002).

[30] A. J. E. M. Janssen, "New analytic results for the Zernike circle polynomials from a basic result in the Nijboer-Zernike diffraction theory", J. Europ. Opt. Soc. Rap. Public. 6, 11028 (2011).

[31] A. J. E. M. Janssen, J. J. M. Braat, and P. Dirksen, "On the computation of the Nijboer-Zernike aberration integrals at arbitrary defocus," J. Mod. Opt. 51, 687-703 (2004).

[32] W. J. Tango, "The circle polynomials of Zernike and their application in optics", Appl. Phys. 13, 327-332 (1977). 